Journal of Mathematics and Statistics 2 (3): 401-406, 2006

ISSN 1549-3644

(C) 2006 Science Publications

\title{
Behavior of the Dedekind's Function over First Order Theta Function According to Conditions Modular Form
}

\author{
İsmet Y1ld1z \\ University of Bahcesehir Vocational School \\ Beşiktaş-İstanbul/TURKEY
}

\begin{abstract}
The effect Dedekind's etha function on theta functions is analyzed according to the characteristics of theta functions under modular group conditions.
\end{abstract}

Key words: Theta functions, characteristic values, modular group, dedekind functions

\section{INTRODUCTION}

By $\mathrm{SL}_{2}$ we mean the group of $2 \times 2$ matrices with determinant 1 . We write $\mathrm{SL}_{2}(\mathrm{R})$ for those elements of $\mathrm{SL}_{2}$ having coefficients in a ring $\mathrm{R}$. In practice, the ring $\mathrm{R}$ will be integers $\mathrm{Z}$, rational numbers $\mathrm{Q}$ and real numbers R. We call $\mathrm{SL}_{2}(\mathrm{Z})$ the modular group $\Gamma$.

If $\mathrm{L}$ is lattice in complex numbers- $\mathrm{C}$, then we can always select a basis, $\mathrm{L}=\left(\omega_{1}, \omega_{2}\right)$ such that $\tau=\frac{\omega_{2}}{\omega_{2}}$ is an element of the upper half-plane $\boldsymbol{\kappa}$, i.e. has $\operatorname{im} \tau \succ 0$ which is not real. If D consist of all $u \in \mathbb{N}$ such that $-\frac{1}{2} \leq \operatorname{Re} u \leq \frac{1}{2}, \quad|u| \geq 1 \quad$ and $\quad \mathrm{T}=\left(\begin{array}{ll}1 & 2 \\ 0 & 1\end{array}\right) \in \Gamma$, $S=\left(\begin{array}{cc}0 & -1 \\ 1 & 0\end{array}\right) \in \Gamma$ then $D$ is a fundamental domain for modular group $\Gamma$ in $\aleph$, Then, $\mathrm{S}, \mathrm{T} \in \Gamma$ generate modular group $\Gamma^{[1]}$. We define characteristic $\left[\begin{array}{c}\varepsilon \\ \mathcal{E}^{\prime}\end{array}\right]$ where $\varepsilon, \varepsilon^{\prime}$ are integers according to characteristics $\left[\begin{array}{l}\varepsilon \\ \varepsilon^{\prime}\end{array}\right] \equiv\left[\begin{array}{l}1 \\ 1\end{array}\right],\left[\begin{array}{l}1 \\ 0\end{array}\right],\left[\begin{array}{l}0 \\ 1\end{array}\right],\left[\begin{array}{l}0 \\ 0\end{array}\right](\bmod 2)$ but $\theta\left[\begin{array}{l}1 \\ 1\end{array}\right](0, \tau) \equiv 0$ for theta function $\theta$. If $\mathrm{n}$ is any positive integer we define $\Gamma_{0}(n)$ to be the set of all matrices $\mathrm{A}=\left(\begin{array}{ll}\alpha & \beta \\ \gamma & \delta\end{array}\right)$ in modular group $\Gamma$ with $\quad \gamma \equiv(\bmod n)$ and but $\Gamma_{0}(n)=\left\{\left(\begin{array}{cc}\alpha & \beta \\ \gamma & \delta\end{array}\right) \in \Gamma(1): \gamma \equiv(\bmod n)\right\}^{[2,3]}$

It is easy to verify that $\Gamma_{0}(n)$ is a subgroup $\Gamma$. If We consider the congruence subgroup $\Gamma(2)$, Then

$\Gamma_{X}(2)=\{W \in \Gamma(1): W \equiv I \quad, \quad W \equiv X(\bmod 2)\}$ where $I$ is the unit matrix and for matrices $X=S, \quad X=T, \quad X=U$ the three subgroups $\Gamma_{S}(2)$,
$\Gamma_{T}(2), \Gamma_{U}(2)$ are conjugate subgroups of $\Gamma(1)$ for $S=\left(\begin{array}{cc}0 & -1 \\ 1 & 0\end{array}\right), T=\left(\begin{array}{ll}1 & 1 \\ 0 & 1\end{array}\right), U=\left(\begin{array}{ll}1 & 0 \\ 1 & 1\end{array}\right), V=\left(\begin{array}{cc}0 & -1 \\ 1 & 1\end{array}\right)$.

We shall need to study such groups when we introduce theta functions.

We note that the above matrices, defined $\mathrm{V}=\mathrm{ST}=$ $\left(\begin{array}{cc}0 & -1 \\ 1 & 1\end{array}\right)$ and $V^{2}=\left(\begin{array}{cc}-1 & -1 \\ 1 & 0\end{array}\right)=\mathrm{I}, \mathrm{U}=\mathrm{TST}$ form a set coset representatives of $\Gamma(1)$ modulo $\Gamma(2)^{[4]}$.

The subgroup $\wp(n)$ of $\Gamma(1)$ is generated by $\mathrm{V}^{2}$ and $\mathrm{S}$ where $\mathrm{k}$ is an odd positive integer and the set of elements in $\wp(k)$ of the form $\left(\begin{array}{ll}\alpha & \beta \\ \gamma & \delta\end{array}\right)$.

Theorem 1: Let $\mathrm{n}$ be any prime and $S \tau=-\frac{1}{\tau}, \quad T \tau=\tau+1$ be the generations of the full modular group $\Gamma$, then for every $W=\left(\begin{array}{ll}a & b \\ c & d\end{array}\right) \in \Gamma$, $W \notin \Gamma_{0}(n)$ there exists an element $K=\left(\begin{array}{ll}p & r \\ s & t\end{array}\right) \in \Gamma_{0}(n)$. Proof: If $W=\left(\begin{array}{ll}a & b \\ c & d\end{array}\right) \in \Gamma$ where $c$ is not $c \equiv 0(\bmod n)$ then we wish to find $W=\left(\begin{array}{ll}a & b \\ c & d\end{array}\right) \in \Gamma$, with $s \equiv 0(\bmod n)$ and an integer $\mathrm{q}, 0 \leq q \leq n$, such that $W=\left(\begin{array}{ll}a & b \\ c & d\end{array}\right)=\left(\begin{array}{ll}\mathrm{p} & \mathrm{r} \\ \mathrm{s} & \mathrm{t}\end{array}\right) S T^{q}$ $=\left(\begin{array}{cc}\mathrm{p} & \mathrm{r} \\ \mathrm{s} & \mathrm{t}\end{array}\right)\left(\begin{array}{cc}0 & -1 \\ 1 & 0\end{array}\right)\left(\begin{array}{ll}1 & \mathrm{q} \\ 0 & 1\end{array}\right)=\left(\begin{array}{cc}\mathrm{p} & \mathrm{r} \\ \mathrm{s} & \mathrm{t}\end{array}\right)\left(\begin{array}{cc}0 & -1 \\ 1 & \mathrm{q}\end{array}\right)$

All matrices here are nonsingular so we can solve for $\left(\begin{array}{ll}\mathrm{p} & \mathrm{r} \\ \mathrm{s} & \mathrm{t}\end{array}\right)$ to get 


$$
\begin{aligned}
& \left(\begin{array}{ll}
p & r \\
s & t
\end{array}\right)=\left(\begin{array}{ll}
\mathrm{a} & \mathrm{b} \\
\mathrm{c} & \mathrm{d}
\end{array}\right)\left(\begin{array}{ll}
0 & -1 \\
1 & \mathrm{w}
\end{array}\right)^{-1} \\
& =\left(\begin{array}{ll}
\mathrm{a} & \mathrm{b} \\
\mathrm{c} & \mathrm{d}
\end{array}\right)\left(\begin{array}{ll}
\mathrm{w} & 1 \\
-1 & 0
\end{array}\right)=\left(\begin{array}{ll}
q a-b & a \\
q c-d & c
\end{array}\right)
\end{aligned}
$$

Choose $\mathrm{q}$ to be that solution of the congruence $\mathrm{qc} \equiv d(\bmod n)$ with $0 \leq q \leq n$. This is possible since $c$ is not $c \equiv 0(\bmod n)$, now take $\mathrm{s}=\mathrm{qs}-\mathrm{t}, \mathrm{p}=\mathrm{wp}-\mathrm{r}, \mathrm{r}=\mathrm{a}$ , $\mathrm{t}=\mathrm{c}$, then $s \equiv 0(\bmod n)$ so $K=\left(\begin{array}{cc}p & r \\ s & t\end{array}\right) \in \Gamma_{0}(n)$.

We define the first order theta function with characteristic $\left[\begin{array}{l}\varepsilon \\ \varepsilon^{\prime}\end{array}\right], u \in C$ and theta period $\tau$ by

$$
\begin{aligned}
& \theta\left[\begin{array}{c}
\varepsilon \\
\varepsilon^{\prime}
\end{array}\right](u, \tau) \\
& =\sum_{N=-\infty}^{\infty} \exp \left\{\left(N+\frac{\varepsilon}{2}\right)^{2} \pi i \tau+2 \pi i\left(N+\frac{\varepsilon}{2}\right)\left(u+\frac{\varepsilon^{\prime}}{2}\right)\right\}
\end{aligned}
$$

where $\mathrm{N}$ is a integer ${ }^{[5]}$.

It has been seen at several points that the theta functions whose characteristics are pair of integers $\left[\begin{array}{c}\varepsilon \\ \varepsilon^{\prime}\end{array}\right]$ satisfy simpler identities than those for which $\varepsilon, \mathcal{E}^{\prime}$ are general real numbers. As $\varepsilon, \mathcal{E}^{\prime}$ are residue classes $(\bmod 2)$ it is natural to concentrate attention on the four functions $\theta\left[\begin{array}{l}1 \\ 1\end{array}\right](u, q), \theta\left[\begin{array}{l}0 \\ 1\end{array}\right](u, q), \theta\left[\begin{array}{l}1 \\ 0\end{array}\right](u, q)$ and $\theta\left[\begin{array}{l}0 \\ 0\end{array}\right](u, q)$ which we shall call the four principal theta functions. For any integers $\mathrm{m}, \mathrm{n}$, when $\mathcal{\varepsilon}, \mathcal{E}^{\prime}$ are integers, we have

$$
\theta\left[\begin{array}{l}
\varepsilon+2 m \\
\varepsilon^{\prime}+2 n
\end{array}\right](u, q)=(-1)^{n \varepsilon} \theta\left[\begin{array}{l}
\varepsilon \\
\varepsilon^{\prime}
\end{array}\right](u, q) \text {. }
$$

When $\varepsilon, \varepsilon^{\prime}$ are integers, the theta series defined by

$$
\theta\left[\begin{array}{l}
\varepsilon \\
\varepsilon^{\prime}
\end{array}\right](u, q)=\sum_{n=-\infty}^{\infty} q^{\left(n+\frac{1}{2}\right)^{2}} e^{2 i\left(n+\frac{1}{2}\right)\left(u-\frac{\varepsilon^{\prime} \pi}{2}\right)}
$$

can be converted into fourier series by pairing off the terms which $n+\frac{\varepsilon}{2}$ has equal and opposite values, $\mathrm{n}$ with $-\mathrm{n}$ if $\varepsilon=0$ and $\mathrm{n}$ with $-(\mathrm{n}+1)$, leaving in the former case an unpaired term for $\mathrm{n}=0$, whose values is 1. The terms so paired have a common factor $q^{\left(n+\frac{\varepsilon}{2)^{2}}\right.}$ and the sum of their remaining factors is $2 \operatorname{Cos}(2 n+\varepsilon)\left(u-\frac{\varepsilon^{\prime} \pi}{2}\right)$. Thus we have the four series $\theta\left[\begin{array}{l}1 \\ 1\end{array}\right](u, q)=2 \sum_{n=-\infty}^{\infty}(-1)^{n} q^{\left(n+\frac{1}{2}\right)^{2}} \operatorname{Sin}(2 n+1) u$

$$
\begin{aligned}
& \theta\left[\begin{array}{l}
1 \\
0
\end{array}\right](u, q)=2 \sum_{n=-\infty}^{\infty} q^{\left(n+\frac{1}{2}\right)^{2}} \operatorname{Cos}(2 n+1) u \\
& \theta\left[\begin{array}{l}
0 \\
1
\end{array}\right](u, q)=1+2 \sum_{n=-\infty}^{\infty}(-1)^{n} q^{n^{2}} \operatorname{Cos} 2 n u \\
& \theta\left[\begin{array}{l}
0 \\
0
\end{array}\right](u, q)=1+2 \sum_{n=-\infty}^{\infty} q^{n^{2}} \operatorname{Cos} 2 n u .
\end{aligned}
$$

Moreover,

$$
\begin{aligned}
& \theta\left[\begin{array}{l}
1 \\
0
\end{array}\right](u)=\theta\left[\begin{array}{l}
1 \\
1
\end{array}\right]\left(u+\frac{\pi}{2}\right) \\
& \theta\left[\begin{array}{l}
0 \\
1
\end{array}\right](u)=-i q^{\frac{1}{4}} e^{i u} \theta\left[\begin{array}{l}
1 \\
1
\end{array}\right]\left(u+\frac{\pi \tau}{2}\right) \\
& \theta\left[\begin{array}{l}
0 \\
0
\end{array}\right](u)=q^{\frac{1}{4}} e^{i u} \theta\left[\begin{array}{l}
1 \\
1
\end{array}\right]\left(u+\frac{\pi+\pi \tau}{2}\right) .
\end{aligned}
$$

If $\mathrm{N}$ is a positive integer then theta function order $\mathrm{n}$ or $\mathrm{n}^{\text {th }}$ is defined by

$$
\theta^{n}\left[\begin{array}{c}
\mu \\
\mu^{\prime}
\end{array}\right](u, \tau)=\sum_{M=-\infty}^{\infty} C_{M} \theta\left[\frac{2\left(M+\frac{\mu}{2}\right.}{N}+(N u, N \tau)\right.
$$

where $0 \leq M \leq N-1$.

In fact, An theta function order $n$ my be found by taking the product of $\mathrm{n}$ first theta functions. Its characteristic $\left[\begin{array}{l}\mu \\ \mu^{\prime}\end{array}\right]$ is given by the matrix sum of the $\mathrm{n}$ characteristic $\left[\begin{array}{c}\varepsilon \\ \varepsilon^{\prime}\end{array}\right]$. The $\mathrm{C}_{\mathrm{M}}$ is indepent on $\mathrm{u}$ put my depent on $\tau$.

$\mathrm{C}_{\mathrm{M}}$ satisfy $\mathrm{C}_{\mathrm{NK}+\mathrm{M}}=\mathrm{C}_{\mathrm{M}} \cdot \exp (\pi i) \cdot \Phi(K)$

where

$\Phi(K)=N \tau\left(K+\frac{\left(M+\frac{\varepsilon}{2}\right)}{N}\right)^{2}+N\left(K+\frac{\left(M+\frac{\varepsilon}{2}\right)}{N}\right) \frac{\varepsilon^{\prime}}{N}$.

Functions $\theta\left[\begin{array}{l}0 \\ 0\end{array}\right](u, \tau)$ has zeros at the points $u=\frac{1}{2}-\frac{1}{2} \tau+r_{1}+r_{2} \tau$. These points form a lattice, that $1-\exp [(2 k-1) \tau+2 u]$ has zeros at points $\mathrm{u}$ where $(2 k-1) \tau \equiv 1(\bmod 2) \quad$ or equivalently. Hence function theta order $n$ defined by

$\Phi(u, \tau)=\prod_{1}^{\infty}\{1+\exp \pi i[(2 k-1) \tau-2 u]\}$

$\prod_{1}^{\infty}\{1+\exp \pi i[(2 k-1) \tau+2 u]\}$ 
has precisely the same zeros as first order theta $\theta\left[\begin{array}{l}0 \\ 0\end{array}\right](u, \tau)$ provided the product converges. Thus we have absolute and uniform convergence of the first infinite product for $\operatorname{im} \tau \succ 0$. For periods 1 and $\tau$, we have

$$
\begin{aligned}
& \Phi(u+1, \tau)=\prod_{1}^{\infty}\{1+\exp \pi i[(2 k-1) \tau+2 u+2]\} \\
& \prod_{1}^{\infty}\{1+\exp \pi i[(2 k-1) \tau-2 u-2]\}=\Phi(u, \tau) \\
& \Phi(u+\tau, \tau)=\prod_{1}^{\infty}\{1+\exp \pi i[(2 k-1) \tau+2 u]\} \\
& \prod_{1}^{\infty}\{1+\exp \pi i[(2 k-1) \tau-2 u]\}
\end{aligned}
$$

Setting $q=\exp \pi i \tau$ we may write

$$
\begin{aligned}
& \Phi(u, \tau)=\prod_{1}^{\infty}\left[1+q^{2 k-1} \exp 2 \pi i u\right] \\
& \prod_{1}^{\infty}\left[1+q^{2 k-1} \exp (-2 \pi i u)\right]
\end{aligned}
$$

It was introduced by Dedekind function $\eta(\tau)$ and is defined by the equation

$$
\eta\left((\tau)=e^{\frac{\pi i \tau}{12}} \coprod_{n=1}^{\infty}\left(1-e^{2 \pi i n \tau}\right) .\right.
$$

The Dedekind function $\eta(\tau)$ is cups form of weight $\frac{1}{2}$ on $\Gamma(1)$ and satisfy

$$
\begin{aligned}
& \eta(A \tau)=v_{\eta}(A)(\gamma \tau+\delta)^{\frac{1}{2}} \eta(\tau) \\
& \text { for all } \mathrm{A}=\left(\begin{array}{ll}
\alpha & \beta \\
\gamma & \delta
\end{array}\right) \in \Gamma(1)^{[6]} .
\end{aligned}
$$

Dedekind proved the following law oh transformation of $\log \eta(\tau)$ under the action of the elliptic modular group. If $\mathrm{A}=\left(\begin{array}{cc}\alpha & \beta \\ \gamma & \delta\end{array}\right) \in \Gamma$ then we have $\log \eta(A(\tau))=\log \eta(\tau)+\frac{1}{12} \pi i \beta \quad$ for $\quad \gamma=0$ and

$\log \eta(A(\tau))=\log \eta(\tau)+\frac{1}{2} \log \left(\frac{\gamma \tau+\delta}{i}\right)$ $\frac{1}{12 \gamma} \pi i(\alpha+\delta)-\pi i g(\gamma, \delta), \quad$ for $\quad c>0$

where $A(\tau)=\frac{\alpha \tau+\beta}{\gamma \tau+\delta}$, all logarithms are taken with respect to the principal branch and $g(\gamma, \delta)$ is a Dedekind sum ${ }^{[7]}$.
An important connection between $\theta\left[\begin{array}{l}0 \\ 0\end{array}\right](0, \tau)$ and $\eta(\tau)$ is given by

$\theta\left[\begin{array}{l}0 \\ 0\end{array}\right](0, \tau) \equiv \eta^{2}\left(\frac{\tau+1}{2}\right) / \eta(\tau+1)$.

The infinite product has the form $\prod\left(1-u^{n}\right)$ where $u=e^{2 \pi i \tau}$. If $\tau \in \aleph$ then $|u| \prec 1$ so the product converges absolutely and non-zero.

Moreover, since the convergence is uniform on compact subsets of $\boldsymbol{\aleph}, \eta(\tau)$ is analytic on $\boldsymbol{\aleph}$. This result and other properties of $\eta(\tau)$ following from transformation formulas which describe the behavior of $\eta(\tau)$ under elements of the modular group $\Gamma$.

i. For the generator $T \tau=\tau+1$ we have $\eta(\tau+1)=e^{\frac{\pi i(\tau+1)}{12}} \coprod_{n=1}^{\infty}\left(1-e^{2 \pi i n(\tau+1)}\right)$

ii. For the other generator $S \tau=-\frac{1}{\tau}$ we have the $\eta\left(-\frac{1}{\tau}\right)=(-i \tau)^{\frac{1}{2}} \cdot \eta(\tau)$.

For proof, let $\tau=i y$ where y $>0$ and then extend the results to all $\tau \in \boldsymbol{\aleph}$ by analytic continuation. The transformation formula becomes $\eta(i / y)=y^{\frac{1}{2}} \eta(i y)$ for $\tau=i y$ and this is equivalent to $\log (i / y)-\log \eta(i y)=\frac{1}{2} \log y$.

and

$\log \eta(i y)=-\frac{1}{12} \pi y+\log \prod_{n=1}^{\infty}\left(1-e^{-2 \pi n y}\right)$

$=-\frac{1}{12} \pi y+\sum_{n=1}^{\infty}\left(1-e^{-2 \pi n y}\right)=-\frac{1}{12} \pi y-\sum_{n=1}^{\infty} \sum_{m=1}^{\infty} \frac{e^{-2 \pi m n y}}{m}$

$=-\frac{1}{12} \pi y-\sum_{m=1}^{\infty} \frac{1}{m}\left(\frac{e^{-2 \pi m y}}{1-e^{2 \pi m y}}\right)$

we obtained $\eta\left(-\frac{1}{\tau}\right)=(-i \tau)^{\frac{1}{2}} \eta(\tau)$ since

$\sum_{m=1}^{\infty} \frac{1}{m}\left(\frac{1}{1-e^{2 \pi m y}}\right)-\sum_{m=1}^{\infty} \frac{1}{m}\left(\frac{1}{1-e^{2 \pi m / y}}\right)_{[2]}$

$-\frac{1}{12} \pi\left(y-\frac{1}{y}\right)=-\frac{1}{2} \log y$

Lemma 1: Let $\Gamma$ be a subgroup of $\Gamma(1)$. If $\varphi(\tau)$ is a modular form of weight for $\Gamma$ with multiplier system $t$ then we write $\varphi(\tau) \in A(\Gamma, n, t)$. If $\varphi(\tau) \in A(\Gamma, n, t)$ then the

$\psi$-transform $\varphi_{\psi}$ of $\varphi$ is defined by

$\varphi_{\psi}(\tau)=\varphi(\tau) / \psi=\{\xi(\psi, \tau)\}^{-1} \varphi(\psi, \tau)$ 
Here, $\xi(\psi, \tau)=(\gamma \tau+\delta)^{n}$ where $A=\left(\begin{array}{ll}* & * \\ \gamma & \delta\end{array}\right)$

If $\varphi_{1}(\tau) \in A\left(\Gamma, n_{1}, t_{1}\right) \quad$ and $\quad \varphi_{2}(\tau) \in A\left(\Gamma, n_{2}, t_{2}\right)$ then we have

$\varphi_{1}(\tau) . \varphi_{2}(\tau) \in A\left(\Gamma, n_{1}+n_{2}, t_{1} . t_{2}\right)$

$\frac{\varphi_{1}(\tau)}{\varphi_{2}(\tau)} \in A\left(\Gamma, n_{1}-n_{2}, \frac{t_{1}}{t_{2}}\right)$

Let $k$ be a prime number greater than 3. If $\sigma$ is a even integer such that $\sigma(k-1) \equiv 0(\operatorname{Mod} 24)$, then $\Theta(\tau)=\left[\frac{\eta(k, \tau)}{\eta(\tau)}\right]^{\rho}$ is a modular function on the group $\Gamma_{0}(k)$. The multiplier system $\rho$ of $\Theta(\tau)$ is given by $\rho(A)=\left[\frac{\delta}{k}\right]^{\rho} \quad$ where $\quad A=\left(\begin{array}{cc}\alpha & \beta \\ \gamma & \delta\end{array}\right) \in \Gamma_{0}(k)$ and $\left[\frac{\delta}{k}\right]$ is Legendre's symbol.

Lemma 2: The functions $\theta\left[\begin{array}{l}0 \\ 1\end{array}\right](0, \tau), \theta\left[\begin{array}{l}0 \\ 0\end{array}\right](0, \tau)$ and $\theta\left[\begin{array}{l}1 \\ 0\end{array}\right](0, \tau)$ are entire modular form of weight $\frac{1}{2}$ for the groups $\Gamma_{S}(2), \Gamma_{T}(2)$ and $\Gamma_{U}(2)$, respectively. Further, $\theta\left[\begin{array}{l}0 \\ 0\end{array}\right](0, \tau) \mid \mathrm{K}=e^{-\frac{\pi i}{4}} \theta\left[\begin{array}{l}0 \\ 1\end{array}\right](0, \tau)$
$\theta\left[\begin{array}{l}0 \\ 0\end{array}\right](0, \tau) \mid \mathrm{K}^{2}=e^{-\frac{\pi i}{2}} \theta\left[\begin{array}{l}1 \\ 0\end{array}\right](0, \tau)$

Also, for $\mathrm{n} \geq 0$

The functions $\theta^{n}\left[\begin{array}{l}0 \\ 1\end{array}\right](0, \tau), \theta^{n}\left[\begin{array}{l}0 \\ 0\end{array}\right](0, \tau)$ and $\theta^{n}\left[\begin{array}{l}1 \\ 0\end{array}\right](0, \tau)$ are entire modular form of weight $\frac{n}{2}$ for the groups $\Gamma_{S}(2), \Gamma_{T}(2)$ and $\Gamma_{U}(2)$, respectively.

Theorem 2: Let $k$ be a prime number greater than 3 and $\sigma$ is a even integer such that $\sigma(k-1) \equiv 0(\operatorname{Mod} 24)$ and put $\mathrm{r}=n \rho$ where $\mathrm{n}$ is a positive integer. If the characteristics $\left[\begin{array}{l}\varepsilon \\ \varepsilon^{\prime}\end{array}\right]$ and $\left[\begin{array}{l}\mu \\ \mu^{\prime}\end{array}\right]$ are $\left[\begin{array}{c}\varepsilon \\ \varepsilon^{\prime}\end{array}\right],\left[\begin{array}{l}\mu \\ \mu^{\prime}\end{array}\right] \equiv\left[\begin{array}{l}1 \\ 0\end{array}\right],\left[\begin{array}{l}0 \\ 1\end{array}\right],\left[\begin{array}{l}0 \\ 0\end{array}\right](\bmod 2)$, the $\phi_{k r}(\tau)$ is a modular function on the group $\Gamma_{0}(k)$. The multiplier system $\rho$ of $\Theta(\tau)$ is given by $\rho(A)=\left[\frac{\delta}{k}\right]^{\rho}$ where $A=\left(\begin{array}{ll}\alpha & \beta \\ \gamma & \delta\end{array}\right) \in \Gamma_{0}(k)$ and $\left[\frac{\delta}{k}\right]$ is Legendre's symbol.
Proof: $\phi_{k r}(\tau) \neq 0$ is regular in $\boldsymbol{\aleph}$. If each positive integers $\sigma, n$ and even positive integer $\mathrm{r}=n \rho$. Therefore, the characteristics $r^{\text {th }}$ order theta functions are $\left[\begin{array}{c}r \varepsilon \\ r \varepsilon^{\prime}\end{array}\right],\left[\begin{array}{c}r \mu \\ r \mu^{\prime}\end{array}\right] \equiv\left[\begin{array}{l}0 \\ 0\end{array}\right](\bmod 2)$, then we have $\phi_{k r}(\tau)=\frac{\theta^{n \rho}\left[\begin{array}{l}\varepsilon \\ \varepsilon^{\prime}\end{array}\right](0, k \tau)}{\theta^{n \rho}\left[\begin{array}{l}\mu \\ \mu^{\prime}\end{array}\right](0, \tau)}=\frac{\theta^{n \rho}\left[\begin{array}{l}0 \\ 0\end{array}\right](0, k \tau)}{\theta^{n \rho}\left[\begin{array}{l}0 \\ 0\end{array}\right](0, \tau)}=$

$$
\left[\frac{\eta^{2}\left(\frac{k \tau+k}{2}\right) / \eta(k \tau+k)}{\eta^{2}\left(\frac{\tau+1}{2}\right) / \eta(\tau+1)}\right]^{n \rho}=\left[\frac{\eta^{2}\left(\frac{k \tau+k}{2}\right) / \eta^{2}\left(\frac{\tau+1}{2}\right)}{\eta^{2}(k \rho) / \eta(\tau+1)}\right]^{n \rho}
$$

Setting $\lambda=\frac{\tau+1}{2} \quad$ and observing that $\Phi(\lambda)=\left[\frac{\eta(k \lambda)}{\eta(\lambda)}\right]^{\rho}$, we obtained $\phi_{k r}(\tau)=\left[\frac{\Phi^{2}(\lambda)}{\Phi^{2}(2 \lambda)}\right]^{n}$ By Lemma1 and from the equation $\Phi(A \tau)=\left[\frac{\delta}{k}\right]^{\rho} . \Phi(\tau)$, we have

$\phi_{k r}(A \tau)=\left[\frac{\Phi^{2}(A \lambda)}{\Phi^{2}(2 \lambda)}\right]^{\rho}=\left[\frac{\left(\frac{\delta}{k}\right)^{2 \rho} \Phi^{2}(\lambda)}{\left(\frac{\delta}{k}\right)^{\rho} \Phi(2 \lambda)}\right]^{n}=\left(\frac{\delta}{k}\right)^{\rho} \phi_{k r}(\tau)$.

Finally, we consider the expansions of $\phi_{k r}(\tau)$ at the parabolic cusp $\infty$ and 0 . Hence, We have

$\Phi(\tau)=\exp \left[\frac{\pi i(k-1) \rho}{12}\right]\left[1+\sum_{N=1}^{\infty} C_{N} \cdot e^{-2 \pi i N \tau}\right] \quad$ as $\quad$ the

Fourier expansion of $\Phi(\lambda)$ function at $\infty . \phi_{k r}(\tau)$ has the Fourier expansion at $\infty$ of the form

$$
\begin{aligned}
& \phi(\tau)=1+\sum_{N=1}^{\infty} C_{N} \cdot e^{2 \pi i N \tau} \\
& \Phi(\tau)=k^{-\frac{\rho}{2}} \exp \left[\frac{\pi i(k-1) \rho}{12 k \tau}\right]\left[1+\sum_{N=1}^{\infty} H_{N} \cdot e^{-2 \pi i N / k \tau}\right]
\end{aligned}
$$

as the Fourier expansion at 0 . Hence

$$
\phi_{k r}(\tau)=\exp \left[\frac{r \pi i(k-1)}{8 k \tau}\right]\left[1+\sum_{N=1}^{\infty} R_{N} \cdot e^{-2 \pi i N / k \tau}\right] .
$$

It follows that $\phi_{k r}(\tau)$ is a modular function on $\Gamma_{0}(k)$.

Theorem 3: $\eta\left(\frac{\alpha \tau+\beta}{\gamma \tau+\delta}\right)=P(\alpha, \beta, \gamma, \delta)\left[-i(\gamma \tau+\delta]^{\frac{1}{2}} \eta(\tau)\right.$ where $\left(\begin{array}{ll}\alpha & \beta \\ \gamma & \delta\end{array}\right) \in \Gamma, \gamma>0$ and

$P(\alpha, \beta, \gamma, \delta)=\exp \left\{\pi i\left[\frac{\alpha+\delta}{12 \gamma}+q(-\delta, \gamma)\right]\right\}$

and 


$$
q(h, k)=\sum_{r=1}^{k-1} \frac{r}{k}\left[\frac{h r}{k}-\left(\frac{h r}{k}\right)-\frac{1}{2}\right]
$$

Note: The sum $q(h, k)$ is called a Dedekind sum .

Theorem 4: The set of modular forms, the entire modular forms and the cups forms each of same dimension for $\Gamma(1)$, form vector space over the complex field.

Let $\mathrm{g}$ be a homogeneous modular form of dimension $-\mathrm{k}$ for the group $\Gamma$ in the variables $\omega_{1}, \omega_{2}$. We write this in the form $g\left[\left(\begin{array}{l}\omega_{1} \\ \omega_{2}\end{array}\right)\right]$ and consider $\left(\begin{array}{l}\omega_{1} \\ \omega_{2}\end{array}\right)$ as a matrix. We define the function $g_{B}$ by

$$
g_{B}\left[\left(\begin{array}{l}
\omega_{1} \\
\omega_{2}
\end{array}\right)\right]=g\left[B\left(\begin{array}{l}
\omega_{1} \\
\omega_{2}
\end{array}\right)\right]
$$

where $M=\left(\begin{array}{ll}\alpha & \beta \\ 0 & \delta\end{array}\right), \alpha>0, \alpha \gamma=\mathrm{n}$ and $i m \frac{\omega_{1}}{\omega_{2}}>0$ and call it a transform of $g$ of order n. It satisfies the following equations

$$
\begin{aligned}
& g_{B}\left[\lambda\left(\begin{array}{l}
\omega_{1} \\
\omega_{2}
\end{array}\right)\right]=\lambda^{-k} \quad g_{B}\left[\left(\begin{array}{l}
\omega_{1} \\
\omega_{2}
\end{array}\right)\right] \text { for } \lambda \in C \quad, \quad \lambda \neq 0 \\
& g_{B}\left[M\left(\begin{array}{l}
\omega_{1} \\
\omega_{2}
\end{array}\right)\right]=g_{B}\left[\left(\begin{array}{l}
\omega_{1} \\
\omega_{2}
\end{array}\right)\right] \text { for } M=\left(\begin{array}{cc}
\alpha & -\beta \\
-\lambda & \delta
\end{array}\right) \in \Gamma_{B} \\
& g_{M B}\left[M\left(\begin{array}{l}
\omega_{1} \\
\omega_{2}
\end{array}\right)\right]=g_{B}\left[\left(\begin{array}{l}
\omega_{1} \\
\omega_{2}
\end{array}\right)\right] \text { for } M=\left(\begin{array}{cc}
\alpha & -\beta \\
-\lambda & \delta
\end{array}\right) \in \Gamma_{B}
\end{aligned}
$$

Therem 5: $\Delta(\tau)=(2 \pi)^{12} \eta^{24}(\tau)=(2 \pi)^{12} x \prod_{n=1}^{\infty}\left(1-x^{n}\right)^{24}$

Proof: Let $f(\tau)=\Delta(\tau) / \eta^{24}(\tau)$. Then $f(\tau+1)=f(\tau)$ and $f\left(-\frac{1}{\tau}\right)=f(\tau)$, so $f$ is invariant under every transformation in $\Gamma$. Also, $f$ is analytic and non-zero in $\boldsymbol{\aleph}$ because $\Delta(\tau)$ is analytic and non-zero and $\eta(\tau)$ never vanishes in $\boldsymbol{\kappa}$. Next we examine the behavior of at $i \infty$. We have

$$
\begin{aligned}
& \eta^{24}(\tau)=e^{2 \pi i \tau} \prod_{n=1}^{\infty}\left(1-e^{2 \pi i n \tau}\right)^{24} \\
& =x \prod_{n=1}^{\infty}\left(1-x^{n}\right)^{24}=x(1+I(x))
\end{aligned}
$$

where $I(x)$ denotes a power series in $x$ with integer coefficients. Thus, $\eta^{24}(\tau)$ has a first order zero at $x=0^{[8]}$.

At first we see the infinite products $\theta\left[\begin{array}{l}0 \\ 0\end{array}\right](u, \tau)$

$$
=\prod_{n=1}^{\infty}\left(1-e^{2 n \pi i \tau}\right) \cdot \prod_{n=1}^{\infty}\left(1+e^{(2 n-1) \pi i \tau+2 \pi i u}\right) \cdot \prod_{n=1}^{\infty}\left(1+e^{(2 n-1) \pi i \tau-2 \pi i u}\right) .
$$

which it converges absolutely.

Theorem 6: We have the relations

$$
\begin{aligned}
& \eta(u)=e^{\frac{\pi i u}{12}} \cdot \theta\left[\begin{array}{l}
0 \\
0
\end{array}\right]\left(\frac{u+1}{2}, 3 u+2 k\right) \\
& \theta\left[\begin{array}{l}
0 \\
1
\end{array}\right]\left(\frac{u+4}{4}, \frac{3}{2} u\right)=e^{-\frac{\pi i u}{12}} \eta(u) \cdot \prod_{n=1}^{\infty}\left(1-e^{(2 n-1) \pi i u}\right)
\end{aligned}
$$

between the functions $\theta\left[\begin{array}{l}0 \\ 0\end{array}\right](u, \tau), \quad \theta\left[\begin{array}{l}0 \\ 1\end{array}\right](u, \tau)$ and Dedekind's $\boldsymbol{\eta}$-function which defined by the infinite product

$\eta(\tau)=e^{\frac{\pi i \tau}{12}} \cdot \prod_{n=1}^{\infty}\left(1-e^{2 n \pi i \tau}\right)$

where $\operatorname{Im} \tau>0$ and $\mathrm{k}$ is a integer.

\section{Proof}

a. Let us recall the formula

$\theta\left[\begin{array}{l}0 \\ 0\end{array}\right](u, \tau)$

$=\prod_{n=1}^{\infty}\left(1-e^{2 n \pi i \tau}\right) \cdot \prod_{n=1}^{\infty}\left(1+e^{(2 n-1) \pi i \tau+2 \pi i u}\right) \cdot \prod_{n=1}^{\infty}\left(1+e^{(2 n-1) \pi i \tau-2 \pi i u}\right)$.

If $\mathrm{k}$ integer, then we have

$\theta\left[\begin{array}{l}0 \\ 0\end{array}\right]\left(\frac{u+1}{2}, 3 u+2 k\right)$

$=\prod_{n=1}^{\infty}\left(1-e^{2 n \pi i(3 u+2 k)}\right) \cdot \prod_{n=1}^{\infty}\left(1+e^{(2 n-1) \pi i(3 u+2 k)+2 \pi i\left(\frac{u+1}{2}\right)}\right) \cdot \prod_{n=1}^{\infty}\left(1+e^{(2 n-1) \pi i(3 u+2 k)-2 \pi i\left(\frac{u+1}{2}\right)}\right)$

$=\prod_{n=1}^{\infty}\left(1-e^{6 n \pi i u}\right) \cdot \prod_{n=1}^{\infty}\left(1+e^{6 n \pi i u-2 \pi i u-(2 k-1) \pi i}\right) \cdot \prod_{n=1}^{\infty}\left(1+e^{6 n \pi i u-4 \pi i u-(2 k+1) \pi i}\right)$

$=\prod_{n=1}^{\infty}\left(1-e^{6 n \pi i u}\right) \cdot \prod_{n=1}^{\infty}\left(1-e^{6 n \pi i u-2 \pi i u}\right) \cdot \prod_{n=1}^{\infty}\left(1-e^{6 n \pi i u-4 \pi i u}\right)$.

If we set $\mathrm{R}=e^{2 \pi i u}$, then we obtain

$\theta\left[\begin{array}{l}0 \\ 0\end{array}\right]\left(\frac{u+1}{2}, 3 u+2 k\right)=\prod_{n=1}^{\infty}\left(1-R^{3 n}\right) \cdot \prod_{n=1}^{\infty}\left(1-R^{3 n-1}\right) \cdot \prod_{n=1}^{\infty}\left(1-R^{3 n-2}\right)$.

On the other hand, we may set $n=n^{\prime}+1$, then

$\theta\left[\begin{array}{l}0 \\ 0\end{array}\right]\left(\frac{u+1}{2}, 3 u+2 k\right)=\prod_{n^{\prime}=1}^{\infty}\left(1-R^{3 n^{\prime}+3}\right) \cdot \prod_{n^{\prime}=1}^{\infty}\left(1-R^{3 n^{\prime}+2}\right) \cdot \prod_{n^{\prime}=1}^{\infty}\left(1-R^{3 n^{\prime}+1}\right)$

$=(1-R)\left(1-R^{2}\right)\left(1-R^{3}\right)\left(1-R^{4}\right) \ldots=$

$\prod_{m=1}^{\infty}\left(1-R^{m}\right)=\prod_{m=1}^{\infty}\left(1-e^{2 m \pi i u}\right)$

According to above, we have $\eta(\tau)=e^{\frac{\pi i \tau}{12}} \cdot \theta\left[\begin{array}{l}0 \\ 0\end{array}\right]\left(\frac{u+1}{2}, 3 u+2 k\right)$

from the Dedekind's $\eta$-function defined by the infinite product 


$$
\eta(\tau)=e^{\frac{\pi i \tau}{12}} \cdot \prod_{n=1}^{\infty}\left(1-e^{2 n \pi i \tau}\right)
$$

where $\mathrm{m}=n^{\prime}$.

b. According to the equation,

$\theta\left[\begin{array}{l}0 \\ 1\end{array}\right](u, \tau)=\sum_{n=-\infty}^{\infty}(-1)^{n} \exp \left(n^{2} \pi i \tau+2 n \pi i u\right)$

we have

$$
\begin{aligned}
& \theta\left[\begin{array}{l}
0 \\
1
\end{array}\right]\left(\frac{u+4}{4}, \frac{3}{2} u\right)=\sum_{n=-\infty}^{\infty}(-1)^{n} \exp \left[\frac{1}{2} n(3 n+1) \pi i u\right] \\
& =1+\sum_{n=1}^{\infty}(-1)^{n}\left\{\exp \left[\frac{1}{2} n(3 n-1) \pi i u\right]+\exp \left[\frac{1}{2} n(3 n+1) \pi i u\right]\right\} \\
& =1+\sum_{n=1}^{\infty}(-1)^{n}\left[x^{\frac{1}{2} n(3 n-1)}+x^{\frac{1}{2} n(3 n+1)}\right]=1- \\
& x-x^{2}+x^{5}+x^{7}-x^{12}-x^{15}+\ldots \\
& \theta\left[\begin{array}{l}
0 \\
1
\end{array}\right]\left(\frac{u+4}{4}, \frac{3}{2} u\right)=(1-x)\left(1-x^{2}\right)\left(1-x^{3}\right) \ldots=\prod_{n=1}^{\infty}\left(1-x^{n}\right)
\end{aligned}
$$

where $x=e^{\pi i u}$ for $|x|<1$, and $\frac{1}{2} n(3 n+1)$ are known as the pentagonal numbers $n=-1,-2, \ldots$

This results play a role of key stone in the forthcoming work concerning relation between the $\theta$ theta function and Dedekind's $\eta$-function. In fact, if the application of theorem(6-b) on the relation obtained with the theorem(-a) which known as the equation between Dedekind's $\eta$-function and L.Euler's theorem on pentagonal numbers is done, we obtain

$$
\begin{aligned}
& \frac{\theta\left[\begin{array}{l}
0 \\
1
\end{array}\right]\left(\frac{u+4}{4}, \frac{3}{2} u\right)}{\prod_{n=1}^{\infty}\left[1-e^{(2 n-1) \pi i \tau}\right]}=\frac{\sum_{n=-\infty}^{\infty}(-1)^{n} \exp \left[\frac{1}{2} n(3 n+1) \pi i u\right]}{\prod_{n=1}^{\infty}\left[1-e^{(2 n-1) \pi i \tau}\right]} \\
= & \frac{\prod_{n=1}^{\infty}\left[1-e^{n \pi i \tau}\right]}{\prod_{n=1}^{\infty}\left[1-e^{(2 n-1) \pi i \tau}\right]}=\prod_{n=1}^{\infty}\left[1-e^{2 n \pi i \tau}\right]=e^{-\frac{\pi i \tau}{12}} \cdot \eta(\tau)
\end{aligned}
$$

As a result, the relation has been obtained between theta and Dedkind's $-\eta(\tau)$ functions by using the characteristic $\left[\begin{array}{l}0 \\ 1\end{array}\right]$ and the variable $\frac{u+4}{4}$ instead of the characteristic $\left[\begin{array}{l}0 \\ 0\end{array}\right]$ and the variable $\frac{u+1}{2}$ which were previously used by Jaccobi.

\section{REFERENCES}

1. İsmet, Y., 2004. On extension of the modular transformations over the modular group by reflection. Appl. Math. Com., 153: 111-116.

2. Kohnen, W., 1985. Fourier coefficients of modular forms of half integral weight. J. Math. Ann., 271: 237-268.

3. Kojima, H., 1980. Cusp forms of weight $\frac{3}{2}$. J. Nagoya Math., 79: 111-122.

4. Brenner, J.L. and R.C. Lyndon, 1983. Maximal nonparabolic subgroups of the modular group. Math. Ann., 263: 1-11.

5. İsmet, Y., 1999. The quadratic relation satisfied by first order theta functions according to quarter periods. J. India Acad. Math., 2: 1.

6. Atiyah, M., 1987. The logarithm of the Dedekind $\eta$-function. J. Math. Ann., 278: 335- 380.

7. Iseki, S., 1957. The transformation formula for the Dedekind modular function and related functional equations. J. Duke Math., 24: 653-662.

8. Van Asch, A.G., 1976. Modular form and root systems. Math.Ann., 222: 145-170. 